\title{
Thermoacoustic Refrigerator Design for Beam Profile Monitor at CERN
}

\section{Holmström, Axi}

IEEE

2019

Holmström , A , Aicheler , M \& Haeggström , E 2019, Thermoacoustic Refrigerator Design for Beam Profile Monitor at CERN . in 2019 IEEE International Ultrasonics Symposium (IUS)

. IEEE International Ultrasonics Symposium , IEEE , pp. 305-308, IEEE International

Ultrasonics Symposium , Glasgow , United Kingdom , 06/10/2019 . https://doi.org/10.1109/ULTSYM.2019.8925816

http://hdl.handle.net/10138/322515

https://doi.org/10.1109/ULTSYM.2019.8925816

unspecified

acceptedVersion

Downloaded from Helda, University of Helsinki institutional repository.

This is an electronic reprint of the original article.

This reprint may differ from the original in pagination and typographic detail.

Please cite the original version. 


\section{Thermoacoustic Refrigerator Design for Beam Profile Monitor at CERN}

\author{
Axi Holmström \\ Electronics research lab., Department of \\ Physics \\ University of Helsinki \\ Helsinki, Finland \\ axi.holmstrom@helsinki.fi
}

\author{
Markus Aicheler \\ Beams department/RF \\ CERN \\ Geneva, Switzerland \\ markus.aicheler@cern.ch \\ Helsinki Institute of Physics \\ Helsinki, Finland
}

\author{
Edward Haeggström \\ Electronics research lab., Department of \\ Physics \\ University of Helsinki \\ Helsinki, Finland \\ edward.haeggstrom@helsinki.fi
}

\begin{abstract}
A travelling-wave thermoacoustic refrigerator design, suitable for CERN's new Ionization Profile Monitor, was devised based on numerical calculations. The design features a Hefilled looped tube containing an acoustic wave produced by an acoustic driver. The parameters of the cooling structure, the regenerator, were constant, whereas the cooling power as a function of tube radius and mean pressure of the gas were investigated. The target, a maximum cold temperature of $273 \mathrm{~K}$ and a minimum cooling power of $12 \mathrm{~W}$, were reached with tube radius $15 \mathrm{~mm}$ at either cold temperature $270 \mathrm{~K}$ and mean pressure $7 \mathrm{~atm}$, or at cold temperature $260 \mathrm{~K}$ and mean pressure $8 \mathrm{~atm}$. The coefficient of performance was also determined.
\end{abstract}

Keywords-Thermoacoustics, cooling, travelling waves

\section{INTRODUCTION}

Thermoacoustic engines are heat engines that either use heat to amplify acoustic waves or use acoustic energy to pump heat, i.e. refrigerate [1]. The basic components of thermoacoustic engines are a fluid-filled (usually a gas) tube containing an acoustic wave and a regenerator/stack - a solid structure containing many narrow channels - with a temperature gradient maintained by heat exchangers. The type of acoustic wave, standing or travelling, and the direction of the temperature gradient determine whether refrigeration or sound amplification occurs. In this paper, a gas-filled travelling-wave thermoacoustic refrigerator (TAR) design is presented.

In a travelling-wave TAR, a travelling wave propagates down the temperature gradient across the regenerator. The regenerator has narrow channels (channel radii smaller than the thermal penetration depth) and needs a much higher specific heat capacity than the gas as well as low thermal conductivity (to maintain the temperature gradient). Heat pumping is produced through a quasi-Stirling cycle at the ends of the regenerator, where the rarefaction phase at the cold end and compression phase at the hot end produce the net heat pumping from cold to hot heat exchanger. The quasi-Stirling cycle of the travelling-wave refrigerator is reversible, unlike the irreversible Brayton cycle of the standing-wave refrigerator. The channel size in a stack inside a standing-wave TAR also needs to be quite

Funding was provided by the Swedish Cultural Foundation in Finland. precise, but the tolerance of the channel size in a travelling-wave TAR's regenerator is large. Due to these reasons, a travellingwave design was chosen.

The application for our TAR is to cool the new Ionization Profile Monitor (IPM) [2] to be installed inside the vacuum of the Proton Synchrotron, a pre-accelerator to the LHC, at CERN, Geneva. The semiconductor chip of the IPM produces $12 \mathrm{~W}$ waste heat during operation and needs to be cooled to $0^{\circ} \mathrm{C}$. Liquid cooling is not desired inside the accelerator since leaks would be disastrous to the accelerator. Peltier elements usually contain Bismuth, which transmutes into Polonium in the radioactive environment of the accelerator [2]. Therefore, a thermoacoustic refrigerator containing gas could provide a suitable cooling solution.

\section{METHODS}

To determine design parameters for the travelling-wave thermoacoustic refrigerator, the model in fig. 1 was used. An acoustic driver, providing the acoustic power with driving frequency $f=50 \mathrm{~Hz}$, is attached to a looped tube containing the regenerator, one heat exchanger on each end (cold and hot heat exchangers) and the thermal buffer tube (TBT) - an insulated tube section attached to the cold heat exchanger where the temperature smoothly approaches the ambient one. The tube

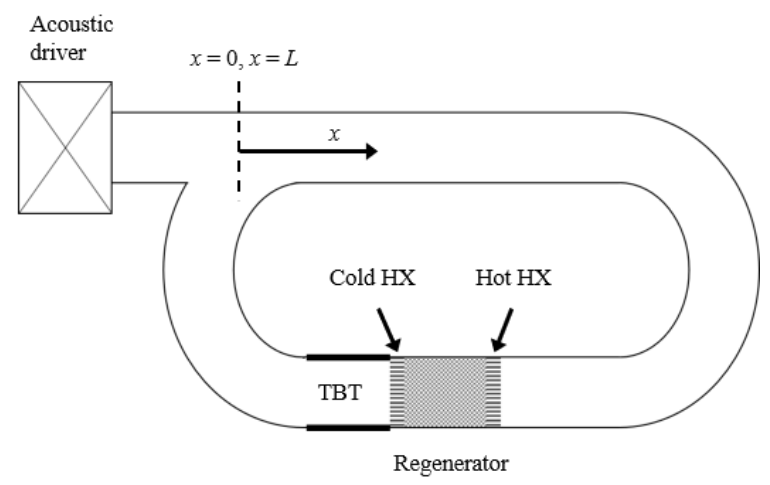

Fig. 1. Schematic of the travelling-wave TAR: the looped tube of length $L$, containing the regenerator, heat exchangers (HX) and thermal buffer tube (TBT). The position $x=0$ is indicated, which is the same as $x=L$. 
contains a travelling wave propagating in the positive $x$ direction and the junction is placed at $x=0$, which is also $x=L$, the length of the looped tube. In these coordinates, the hot heat exchanger precedes the regenerator and the cold heat exchanger succeeds it, followed by the TBT. An ambient temperature of $T_{0}=290 \mathrm{~K}$ was chosen. Cold temperatures, $T_{C}$, were either $270 \mathrm{~K}$ or $260 \mathrm{~K}$.

\section{A. Model parameters}

Helium was chosen as the working gas inside the tube. The thermal dependence of both the viscosity, $v$, and thermal conductivity, $k$, of the gas in different TAR sections were considered. Temperatures were assigned as follows: ambient temperature, $T_{0}$, in the tube sections and hot heat exchanger, average temperature, $T_{m}=\left(T_{0}+T_{C}\right) / 2$, in the regenerator and TBT, and cold temperature, $T_{C}$, in the cold heat exchanger. The mean pressure, $p_{m}$, of the gas was varied from $5 \mathrm{~atm}$ to $10 \mathrm{~atm}$. Temperature and pressure dependences of the adiabatic sound speed and density of the gas were accounted for using the ideal gas law. This ensured that the temperature and pressure dependences of the viscous and thermal penetration depths, $\delta_{v}$ and $\delta_{k}$, respectively, and by extension the Prandtl number, $\sigma=\left(\delta_{v} / \delta_{k}\right)^{2}$, were taken into account. The pressure amplitude of the wave at $x=0$ was set to $p_{0}=0.05 \cdot p_{m}$, as coupling a higher amplitude wave into a gas is difficult.

Constant absolute or relative design parameters are summarized in Table 1 . The tube was selected to be $L=0.05 \cdot \lambda$ long, with $\lambda$ being the adiabatic wavelength in the gas. The tube radius was either fixed at $r_{\text {tube }}=15 \mathrm{~mm}$ or varied from $14.4 \mathrm{~mm}$ to $20 \mathrm{~mm}$. The regenerator was chosen to be $5 \mathrm{~cm}$ long and to comprise circular channels with radius $r=150 \mu \mathrm{m}$. This channel radius was chosen because materials with this pore size are available and it fulfills the criterion $\left(r / \delta_{k}\right)^{2}<3$ (this ratio was in the range 0.11 to 0.23 , the variation is caused by its pressure

TABLE I. DESIGN PARAMETERS

\begin{tabular}{|l|l|}
\hline \multicolumn{2}{|l|}{ Constant Absolute or Relative Design Parameters } \\
\hline Parameter & Value \\
\hline Ambient temperature $T_{0}$ & $290 \mathrm{~K}$ \\
\hline Pressure amplitude $p_{0}$ & $0.05 \cdot p_{m}$ \\
\hline Frequency $f$ & $50 \mathrm{~Hz}$ \\
\hline Tube length $L$ & $0.05 \cdot \lambda=1 \mathrm{~m}$ \\
\hline Thermal buffer tube length $L_{\text {tbt }}$ & $0.005 \cdot \lambda=0.1 \mathrm{~m}$ \\
\hline Regenerator & \\
\hline Material & Stainless steel \\
\hline Porosity & 0.54 \\
\hline Channel radius $r$ & $150 \mu \mathrm{m}$ \\
\hline Position $x_{\text {reg }}$ & $0.8 \cdot L=0.8 \mathrm{~m}$ \\
\hline Regenerator length $L_{r e g}$ & $5 \mathrm{~cm}$ \\
\hline Heat exchangers & \\
\hline Material & Brass \\
\hline Length $L_{H X}$ & $5 \mathrm{~mm}$ \\
\hline Plate thickness & $0.5 \mathrm{~mm}$ \\
\hline Plate spacing & $1 \mathrm{~mm}$ \\
\hline
\end{tabular}

dependence) [3]. The regenerator was positioned at $x_{\text {reg }}=0.8 \cdot \mathrm{L}$ [4]. The identical heat exchangers were modelled after those used by Bassem et al. [5]. The length of the thermal buffer tube was set to $L_{t b t}=0.005 \cdot \lambda$. The tube length, $L$, the relative position of the regenerator, $x_{\text {reg }} / L$, and the length of the TBT, $L_{t b t}$, were selected based on calculations performed by Ueda et al. [4]. With our parameters, these lengths were $L=1.0 \mathrm{~m}, L_{t b t}=10 \mathrm{~cm}$ and $x_{\text {reg }}=80 \mathrm{~cm}$, respectively.

\section{B. Calculation method}

The calculations are based on Rott's thermoacoustic approximation [6], here expressed as the thermoacoustic versions of the momentum and continuity equations used by Swift [1]:

$$
\begin{gathered}
\frac{d p}{d x}=-\frac{i \omega \rho_{m}}{A\left(1-f_{v}\right)} U \\
\frac{d U}{d x}=-\frac{i \omega A\left[1+(\gamma-1) f_{k}\right]}{\gamma p_{m}} p+\frac{f_{k}-f_{v}}{\left(1-f_{v}\right)(1-\sigma)} \frac{1}{T_{m}} \frac{d T_{m}}{d x} U
\end{gathered}
$$

where $p$ is pressure, $U$ volumetric velocity, $\omega$ angular frequency, $\rho_{m}$ mean density, $A$ cross-sectional area of the tube/channel, $\gamma$ heat capacity ratio, $d T_{m} / d x$ temperature gradient and $i$ the imaginary unit. The complex functions $f_{k}$ and $f_{v}$ are spatial averages describing the thermal and viscous interactions, respectively, for a certain channel geometry [6]. A transfer matrix describing the change in pressure and volumetric velocity can be constructed for each tube section using these equations $[3,7]$.

Two operating conditions must be met. First, the pressure $p$ must be continuous at the junction, i.e. $p(x=0)=p(x=L)$. Second, the total power, $H$, (the sum of the enthalpy flow and heat conduction) must be constant throughout the regenerator and thermal buffer tube, respectively. The total power $H$ is [8]:

$$
\begin{gathered}
H=\frac{1}{2} \operatorname{Re}\left[p \widetilde{U}\left(1-\frac{f_{k}-\tilde{f}_{v}}{(1+\sigma)\left(1-\tilde{f}_{v}\right)}\right)\right]+\frac{\rho_{m} c_{p}|U|^{2} \operatorname{Im}\left[f_{k}+\sigma \tilde{f}_{v}\right]}{2 A \omega\left(1-\sigma^{2}\right)\left|1-f_{v}\right|^{2}} \frac{d T_{m}}{d x} \\
-\left(A k+A_{\text {sol }} k_{\text {sol }}\right) \frac{d T_{m}}{d x}
\end{gathered}
$$

$c_{p}$ is the isobaric heat capacity of the gas, $A$ and $A_{\text {sol }}$ the crosssectional areas of the gas and solid in the regenerator, respectively, and $k$ and $k_{\text {sol }}$ the thermal conductivities in the gas and solid, respectively. Streaming was neglected.

By assuming an initial pressure $p_{0}$ at $x=0$ and by using the iterative procedure described in detail in [3, 7], these two conditions are satisfied and the pressure and volumetric velocity distributions in the entire tube and the total power and temperature distributions in the regenerator and TBT are obtained.

Knowing the pressure, volumetric velocity, and total power distributions, the cooling power can be obtained by calculating the power flows shown in fig. 2 . The acoustic power, $W$, is:

$$
W=\frac{1}{2} \operatorname{Re}[p \widetilde{U}]
$$

The heat flow inside the regenerator and thermal buffer tube are:

$$
Q=H-W
$$


At the cold heat exchanger, sandwiched between the thermally insulated regenerator and TBT, heat flows both from the cold end of the regenerator toward the hot end and from the TBT toward the cold heat exchanger. The absolute difference between these heat flows is the cooling power, $Q_{C}$, of the refrigerator, as indicated in fig. 2. The coefficient of performance, $C O P$, is then obtained as the ratio of cooling power $Q_{C}$ to the acoustic power consumed in the device $\Delta W_{\text {tot }}$ :

$$
C O P=Q_{C} / \Delta W_{t o t}
$$

where $\Delta W_{\text {tot }}$ is the difference in acoustic power at the beginning and end of the looped tube, i.e. $\Delta W_{t o t}=W(x=0)-W(x=L)$. The $C O P$ can be compared to the ideal theoretical limit, the Carnot coefficient of performance, $C O P_{\text {Carnot }}=T_{C} /\left(T_{0}-T_{C}\right)$.

To elucidate the behavior of the $C O P$, it is also useful to consider the heat pumping efficiency of the regenerator, $\eta$, and the dimensionless dissipation outside the regenerator, $\Gamma$ [4]. The COP depends on these quantities as follows:

$$
\begin{gathered}
C O P=C O P_{\text {Carnot }} \cdot \eta \cdot(1-\Gamma), \\
\eta=\left(Q_{C} / \Delta W_{\text {reg }}\right) / C O P_{\text {Carnot }} \\
\Gamma=\left(\Delta W_{\text {tot }}-\Delta W_{\text {reg }}\right) / \Delta W_{\text {tot }}
\end{gathered}
$$

where $\Delta W_{\text {reg }}$ is the acoustic power consumed in the regenerator.

\section{RESULTS}

Two parameters were varied independently to explore when the desired cooling power of $12 \mathrm{~W}$ could be reached: the tube radius and the mean pressure. The tube radius was chosen because a larger tube allows a greater number of narrow channels in the regenerator, which produce the cooling. While the pressure amplitude is more crucial to the cooling power than the mean pressure $p_{m}$, it was assumed that a wave of higher amplitude than $0.05 \cdot p_{m}$ would be difficult to produce, and therefore the mean pressure was chosen as the second variable.

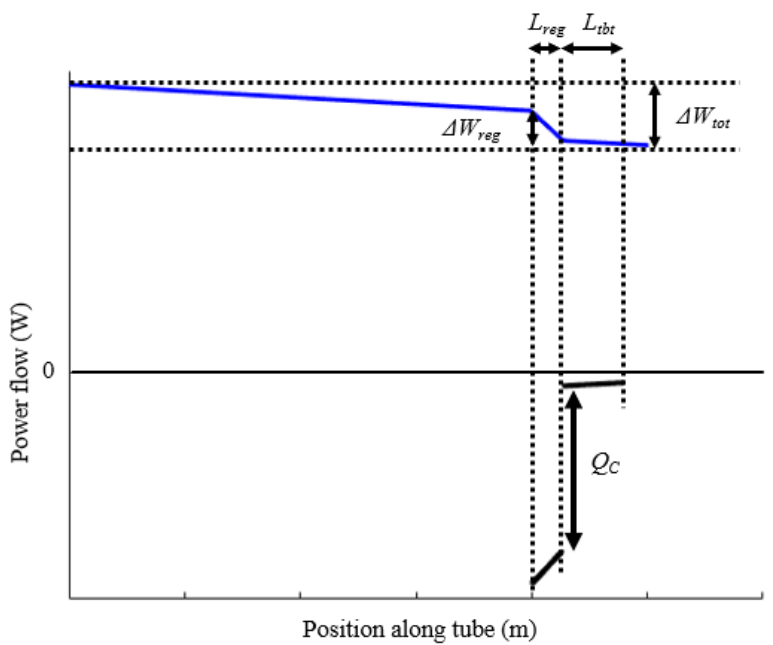

Fig. 2. Power flows along the TAR. The blue line indicates acoustic power, the black lines heat flows in the regenerator and TBT. Acoustic power consumed in the refrigerator, $\Delta W_{t o t}$, acoustic power consumed in the regenerator, $\Delta W_{\text {reg }}$, and the cooling power $Q_{C}$ are indicated.
The calculated cooling power as a function of tube radius and mean pressure is shown in fig. $3 \mathrm{a} \& \mathrm{~b}$, respectively. When the tube radius was varied, the mean pressure was kept at $p_{m}=7 \mathrm{~atm}$ and the cold temperature at $T_{C}=270 \mathrm{~K}$. As shown in fig. 3a, a cooling power of $12 \mathrm{~W}$ was reached at a tube radius $r_{\text {tube }}=15 \mathrm{~mm}$. Further increasing the tube radius increases the cooling power as the regenerator can fit more narrow channels inside it. Next, the tube radius was fixed at $r_{\text {tube }}=15 \mathrm{~mm}$ and the mean pressure was varied (fig. $3 \mathrm{~b}$ ). As the maximum acceptable cold temperature was $T_{C}=273 \mathrm{~K}$, the cold temperature was changed to $T_{C}=260 \mathrm{~K}$ to have a larger temperature margin. Fig. $3 \mathrm{~b}$ shows that decreasing the cold temperature raises the $12 \mathrm{~W}$ cooling power limit from $p_{m}=7$ atm to $p_{m}=8 \mathrm{~atm}$.

In both cases (varying tube radius and mean pressure), the coefficient of performance, $C O P$, heat pumping efficiency, $\eta$, and dimensionless dissipation outside the regenerator, $\Gamma$, were calculated (fig. 4). The theoretical limit, the Carnot $C O P$, was $C O P_{\text {Carnot }}=13.5$ when tube radius was varied and $C O P_{\text {carnot }}=8.7$ when mean pressure was varied. At the $12 \mathrm{~W}$ target, the $C O P$ reached $0.22 \cdot \mathrm{COP}_{\text {Carnot }}$ and $0.29 \cdot \mathrm{COP}_{\text {Carnot }}$ when tube radius and mean pressure were varied, respectively.

The heat pumping efficiency, $\eta$, is little influenced by the small change in tube radius (fig. 4 a), which is understandable as increasing the tube radius does increase the number of channels in the regenerator, but the loss in each channel remains approximately the same. Increasing the mean pressure, however, has an impact (fig. 4 b). The higher pressure decreases the viscous and thermal penetration depths, which causes the total power flow $H$ through the regenerator to decrease, thereby increasing the cooling power, according to (5). The acoustic power consumed in the regenerator, $\Delta W_{\text {reg }}$, also decreases as the viscous losses decrease. These effects combined increase the

a)

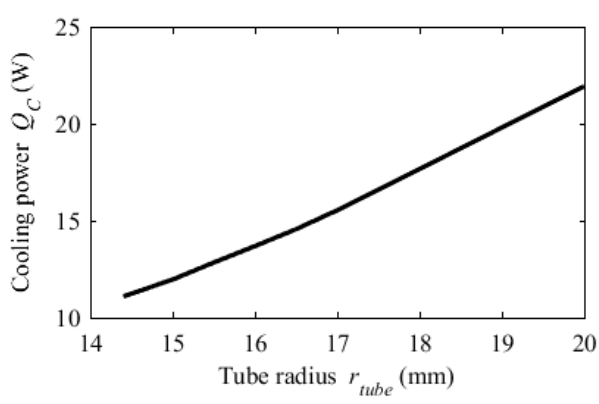

b)

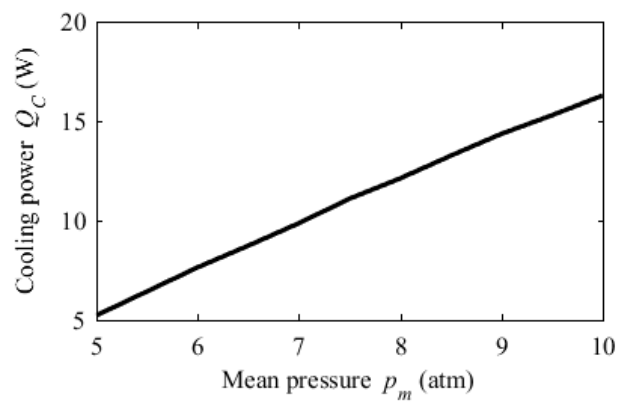

Fig. 3. Cooling power $Q_{C}$ as a function of a) tube radius at $270 \mathrm{~K}$ cold temperature and b) mean pressure at $260 \mathrm{~K}$ cold temperature. 
a)

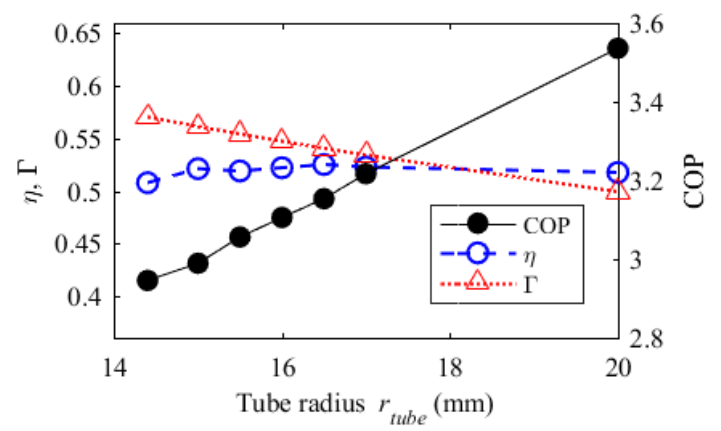

b)

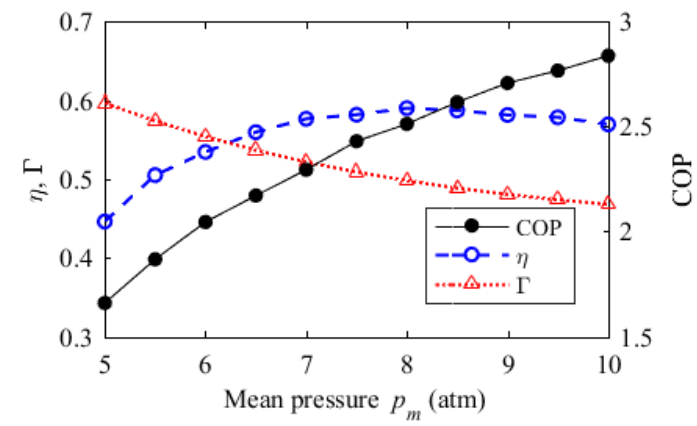

Fig. 4. Coefficient of performance, $C O P$, heat pumping efficiency, $\eta$, and dimensionless dissipation outside the regenerator, $\Gamma$, as functions of a) tube radius and $\mathrm{b}$ ) mean pressure. Cold temperatures were $270 \mathrm{~K}$ and $260 \mathrm{~K}$ in a) and $b)$, respectively.

heat pumping efficiency, as defined in (8). Dimensionless dissipation inside the tube decreases both with a larger tube radius and higher mean pressure (fig 4 a \& b), which in both cases can be explained by a decrease in viscous losses.

\section{DISCUSSION}

Simulation tools specifically designed for thermoacoustics exist, e.g. Delta EC [9], but they have the disadvantage that the user must define the acoustic impedance for some component of the device. If the device contains a distinct end (a tube end or compliance) this can be done, but in a looped tube this is not straightforward. The transfer matrix method used here and described in detail by Ueda et al. [3, 7] only requires an assumption of initial pressure $p_{0}$ at $x=0$. Then again, the transfer matrix method requires recalculation of the temperature distributions in an iterative process, and as the total power $H$ is not initially known, neither in the regenerator nor in the TBT, it requires parametrizations of these to obtain temperature distributions corresponding to the selected hot and cold temperatures.

Many sources of losses in the TAR were considered in this study: viscous and thermal losses of the acoustic wave and the heat conduction in both the Helium and the regenerator's solid structure. However, streaming was neglected, as were losses at the heat exchangers (i.e. the heat exchangers were assumed to be in perfect contact with the regenerator) and losses caused by possible thermal conduction along the tube casing containing the regenerator. Further parameters that could be optimized in the calculations are frequency (affects the thermal and viscous contact), channel radius in the regenerator (now fixed to readily available materials) and the position of the regenerator inside the tube. Still, utilizing the presented results and selecting a tube radius and mean pressure that yielded a cooling power higher than $12 \mathrm{~W}$ should ensure sufficient cooling and cold temperature.

\section{REFERENCES}

[1] G. W. Swift, Thermoacoustics: A Unifying Perspective for Some Engines and Refrigerators, 2nd ed. Springer International Publishing, 2017.

[2] D. Bodart et al., "Development of an ionization profile monitor based on a pixel detector for the CERN Proton Synchrotron," in Proceedings of IBIC 2015, Melbourne, Australia, 2015, pp. 13-17.

[3] Y. Ueda and C. Kato, "Stability analysis of thermally induced spontaneous gas oscillations in straight and looped tubes," J. Acoust. Soc. Am., vol. 124, no. 2, pp. 851-858, 2008.

[4] Y. Ueda, B. M. Mehdi, K. Tsuji, and A. Akisawa, "Optimization of the regenerator of a traveling-wave thermoacoustic refrigerator," J. Appl. Phys., vol. 107, no. 3, p. 034901, 2010.

[5] M. Bassem, Y. Ueda, and A. Akisawa, "Design and construction of a traveling wave thermoacoustic refrigerator," Int. J. Refrig., vol. 34, no. 4, pp. 1125-1131, 2011

[6] N. Rott, "Damped and thermally driven acoustic oscillations in wide and narrow tubes," Z. Angew. Math. Phys., vol. 20, no. 2, pp. 230-243, 1969.

[7] Y. Ueda, "Calculation method for the prediction of the performance of a traveling-wave thermoacoustic cooler," Journal of Power and Energy Systems, vol. 2, no. 5, pp. 1276-1282, 2008.

[8] N. Rott, "Thermally driven acoustic oscillations, part III: Second-order heat flux," Z. Angew. Math. Phys., vol. 26, no. 1, pp. 43-49, 1975.

[9] Delta EC, Los Alamos National Laboratory. Available: https://www.lanl.gov/org/ddste/aldps/materials-physicsapplications/condensed-matter-magnetscience/thermoacoustics/computer-codes.php. (Accessed 30.8.2019). 